\title{
Pain Sensation: Infrequent Case of Root Resorption of the Lower Second Molar Root with Bone Regeneration after Impacted Third Molar Removal
}

\author{
Chandrashekhar Pandey ${ }^{1, \odot}$ \\ Natthamet Wongsirichat ${ }^{1}$, \\ Bishwa Prakash Bhattarai ${ }^{1, \odot}$ \\ Suphachai Suphangul ${ }^{4}$, \\ ${ }^{1}$ Clinical Science Department, International College of Dentistry, \\ Walailak University, Phayathai District, Bangkok, Thailand \\ ${ }^{2}$ Department of Oral and Maxillofacial Surgery, Faculty of Dentistry, \\ Mahidol University, Ratchathewi District, Bangkok, Thailand \\ ${ }^{3}$ Department of Special Clinic, Private practice in Faculty of \\ Dentistry Mahidol University, Ratchathewi District, Bangkok, \\ Thailand \\ ${ }^{4}$ Department of Advanced General Dentistry Faculty of Dentistry, \\ Mahidol University, Ratchathewi District, Bangkok, Thailand
}

\section{Manop Khanijou ${ }^{2,3, \odot ~ P i m o l m a s ~ N a ~ S o n g k l a ~}{ }^{3, \odot}$}

Address for correspondence Suphachai Suphangul, DDS, Grad. Dip. In Clin. Sc. (Oral \& Maxillofacial Surgery), FRCDS (Thailand), Department of Advanced General Dentistry Faculty of Dentistry, Mahidol University, 6 Yothi Street Ratchathewi District, Bangkok 10400, Thailand (e-mail: kungomfs@gmail.com).

\begin{abstract}
Keywords

- external root resorption

- pressure resorption

- tooth impaction

- intra-oral peri-apical radiograph

- vitality test

There have been infrequent case reports of bilateral root resorption in the lower second molar secondary from impacted third molar removal with bone regeneration without pain. Root resorption is commonly observed during third molar surgical impaction that affects mandibular second molars. The bone regeneration into the adjacent affected tooth is evident simply after the surgical extraction of the impacted tooth. However, there have been a few previous reports on the prognosis of the remaining resorbed tooth. Most dentists often choose to extract them when damaged if root resorption is observed. This report case involved bilateral root resorption of the lower second molar due to lower adjacent impacted third molar teeth and bone regeneration without pain sensation. After surgical extraction of bilateral lower impacted third molar teeth, the remaining teeth retained vital pulp and survived as functional teeth.
\end{abstract}

\section{Introduction}

Third molars are the last permanent teeth to erupt. The absence of adequate space, late development of roots, physical bone barrier in the line of eruption lead to impaction (lower impacted third molars: LITM). This impacted condition may cause several pathological conditions including carious lesions in the adjacent tooth, pericoronitis, periodontal conditions, odontogenic or dentigerous cysts, and tumors and external root resorption (ERR) of second molars. ${ }^{1-3}$

ERR of lower second molars, considered as pressure resorption, is a pathological process caused by LITM, the

DOI https://doi.org/ $10.1055 / \mathrm{s}-0041-1735763$ ISSN 2320-4753 action of orthodontic appliances, chronic periapical periodontitis, dental trauma, cysts, and tumors. The incidence of ERR in lower second molars caused by LITM is not prevalent as cited in several previous studies ${ }^{4}$ wherein its incidence varies from $3.05 \%$ to $24.2 \% .5$

In many cases, following surgical removal, the pain from surgery could be misleading causing misdiagnosis and hence could cause extraction of the adjacent resorbed tooth. However in our study, after proper management of pain related to surgical removal of impacted, the adjacent resorbed tooth was under specialist endodontist observation and no treatment was given because the patient was 
asymptomatic. $^{7}$ As depicted in many studies by Israel HA et al. misdiagnosis and multiple failed treatments could be dangerous to the patient since tooth extraction is a nonreversible treatment and could increase the cost of future tooth replacement therapy. ${ }^{8}$

Once diagnosed, the ERR of lower second molars is often treated by removing the pressure-causing factor as LITM. $^{9}$ There have been a few previous reports on the prognosis of the remaining resorbed tooth, as extraction is often indicated in those conditions. This article reports a case of bilateral resorption of the lower second root due to LITM and secondary bone regeneration after surgical removal of bilateral LITM.

\section{Case Report}

A 25-year-old male patient diagnosed with ERR of 37, 47 due to post-surgical extraction of 38,48 was referred to the Department of Oral and Maxillofacial Surgery Clinic, Faculty of Dentistry, Mahidol University for the treatment. The surgical removal of 38 and 48 was done at the Department of Oral and Maxillofacial Surgery, Faculty of Dentistry, Mahidol University with a 4-weeks' washout period between first and second operations (-Fig. 1).

The patient returned for the follow-up of 37,47 oral hygiene, and complained regarding cheek biting of 38,48 regions on both sides.

The patient was asymptomatic when he visited the Department of Oral and Maxillofacial Surgery. Clinical examination of 37 and 38 showed no mobility with sound gingival and periodontal condition (color, texture, and margins). Regions 37, 38 showed normal response to the vitality test (electric pulp test) and thermal test (cold test), and had no tenderness on percussion.

Intra-oral periapical (IOPA) radiograph of region 37 revealed resorption of the distal root of 37 at the cervical third, extending from cementoenamel junction to the distocoronal one-third of the root and distal root canals. IOPA also showed radio-opacity in real-time extraction socket of region 38, without any periapical and periradicular pathology. Similar changes were observed in the root of 47 , periradicular areas as observed in the IOPA ( - Figs. 2 and $\mathbf{3}$ ).

The case was diagnosed as bilateral ERR in real time 37, 38 with normal vital pulp and apical tissues.

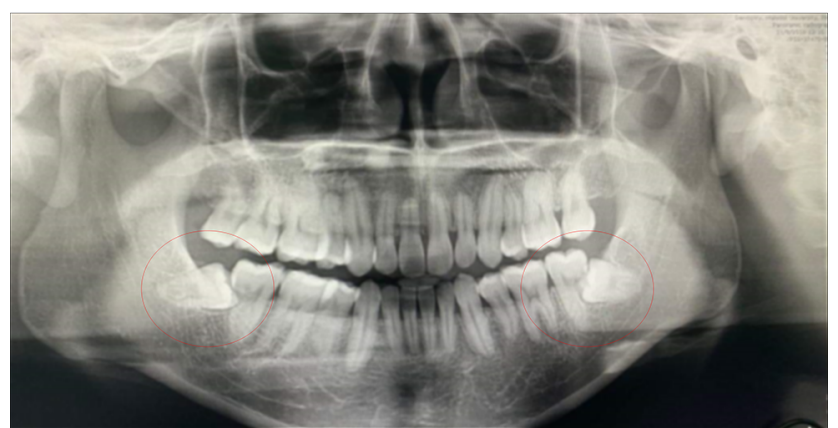

Fig. 1 Pre-operation of impacted 38, 48 (circles).

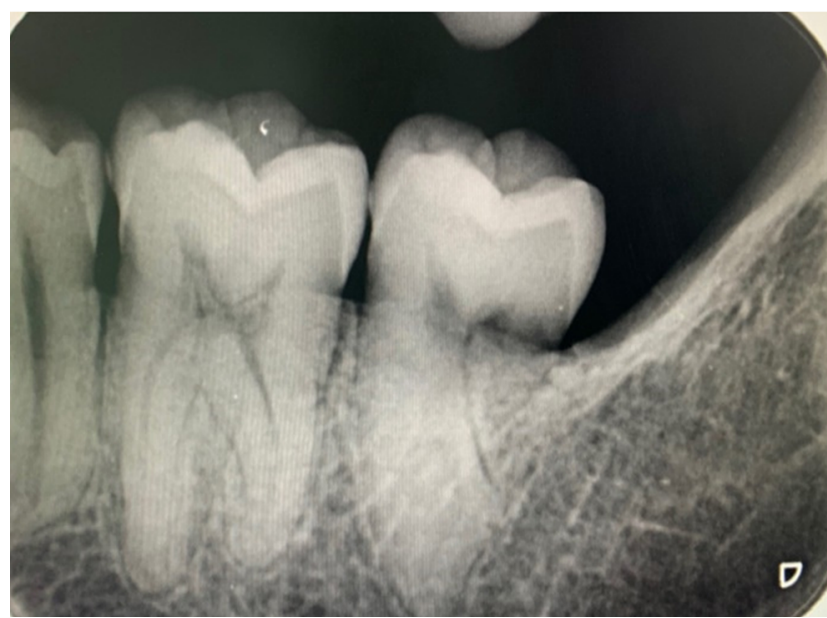

Fig. 2 External root resorption in real-time 37.

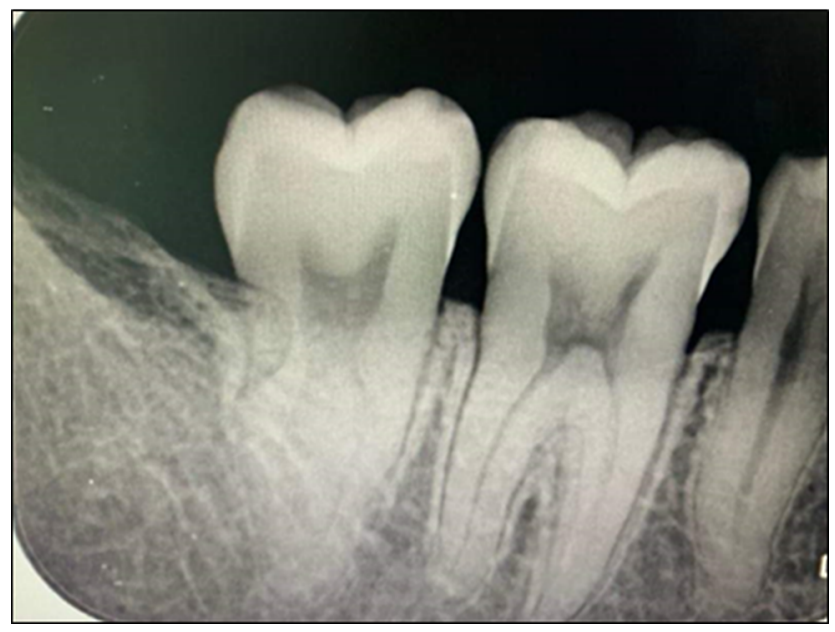

Fig. 3 External root resorption in real-time 47.
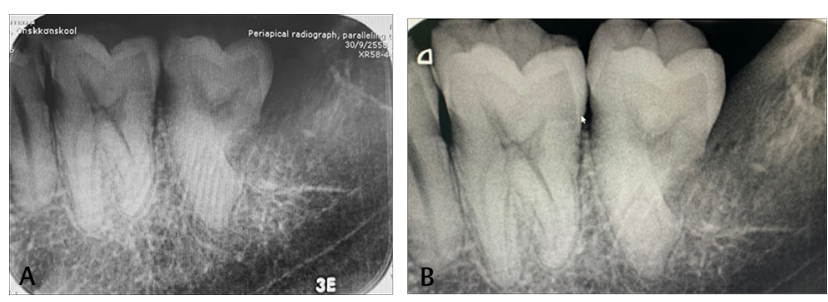

Fig. 4 (A, B) 37 at 3 months and 6 months follow-ups.

Observing the normal status of tooth and periodontium, follow-ups were advised without any extraction intervention. The patient was recalled after 3 months' and 6 months' intervals after the first visit. Observations after the third and sixth months were similar. The patient was asymptomatic with no mobility, the tooth was fully functional, and normal results were obtained from vitality and percussion tests. The IOPA X-ray showed bone regeneration in the root canal of both teeth with a healing extraction socket of 38, 48 and relatively narrow root canals (- Figs. 4A, B and 5A, B). 

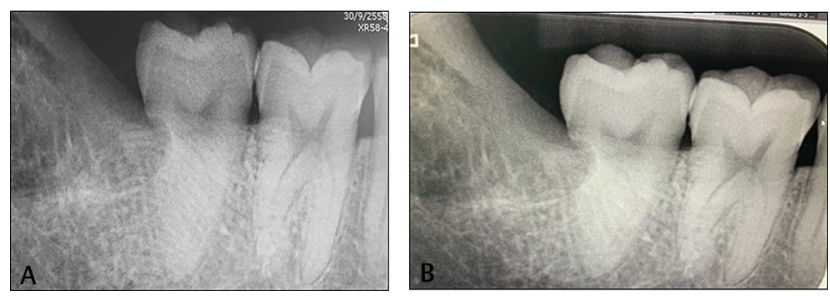

Fig. 5 (A, B) 47 at 3 months and 6 months follow-ups.

\section{Discussion}

ERR in lower second molars may be caused by third molar impaction as observed in this case. The prevalence has been found to vary from $0.9 \%$ to $16 \% .^{9,10}$ The studies using CBCT have shown higher occurrence as compared with panoramic radiographs, varying from $20.17 \%$ to $74 \% .^{9}$

The case was diagnosed by IOPA, tooth 37,47 with deep resorption, and bone regeneration of both teeth. We referred the patient to the endodontist for the treatment of region 37 , 47. However, the endodontist informed the patient that the teeth did not require treatment because there were no symptoms or complications.

The majority of the ERR case occurred in the mandibular molars. This finding is consistent with various studies in which ERR was found more prevalent in the mandible. ${ }^{11}$ This can be related to the anatomical characteristics of the mandible and the lack of mandibular arch space leading to LITM. This case showed the occurrence of ERR in the cervical third of the tooth, which is consistent with many previous studies reporting similar occurrences. ${ }^{12}$

The root complication condition of this case occurred from mechanical forces during the eruption of LITM. Pressure resorption is related to the pathological process activating from the resorptive cells. ${ }^{13}$ The conditions had no peri-radicular pathology, periapical or periodontal anomaly with normal vitality and percussion tests results, and bone regeneration. These findings led to the decision of eliminating tooth extraction as a treatment option for the ERR. The prognosis after follow-up for both teeth was satisfactory.

\section{Conclusion}

The extraction of patients' second molar tooth with ERR secondary to third molar impaction has been practiced for decades. However, as dental health professionals, conserving healthy and natural dentition should always be a priority. This rare case report presented the management of similar cases of bilateral ERR that showed a good prognosis post-surgical removal of the impacted tooth without affecting tooth vitality. In cases, where patients are asymptomatic, mere observation of resorbed tooth could be included in the treatment plan; moving forward, dentists should carefully assess the teeth before deciding the treatment plan for ERR of lower second molar cases affected by third molar impaction. Future studies would involve a thorough CT scan to study the extent of bone regeneration into the adjacent resorbed tooth.

\section{Authors' Contributions}

Natthamet Wongsirichat contributed in the conceptualization, supervision, and the final approval of the article. Suphachai Suphangul contributed in the conceptualization and methodology of the article. Chandrashekhar Pandey contributed in the methodology, formal analysis, and writing the original draft of the article. Manop Khanijou contributed in the validation, investigation, visualization, and project administration of the article. Pimolmas $\mathrm{Na}$ Songkla contributed with the resources. Bishwa Prakash Bhattarai contributed in data curation and reviewing and editing the article. Suphachai Suphangul and Manop Khanijou provided the agreement to accountability for the article.

\section{Declaration of Patient Consent}

The authors certify that they have obtained all appropriate patient consent forms. In the form, the patient(s) has/have given his/her/their consent for his/her/their images and other clinical information to be reported in the journal. The patients understand that their names and initials will not be published and due efforts will be made to conceal their identity, but anonymity cannot be guaranteed.

\section{Ethics Approval}

This case report required no approval by the institutional review board of Mahidol University. However, we informed the patient and received the signed consent.

\section{Conflicts of Interest}

The authors declare no conflict of interest.

\section{Acknowledgments}

The authors would like to thank the staff and dental assistants including colleagues and coworkers in the Department of Oral and Maxillofacial Surgery, Faculty of Dentistry, Mahidol University, Bangkok, Thailand. Finally, the authors would like to thank Christian Estacio for editing and revising the language of this manuscript.

\section{References}

1 Armas JM, Savarrio L, Brocklebank LM. External apical root resorption: two case reports. IntEndodJ2008;41(11):997-1004

2 Li ZB, Qu HL, Zhou LN, Tian BM, Gao LN, Chen FM. Nonimpacted third molars affect the periodontal status of adjacent teeth: a cross-sectional study. J Oral Maxillofac Surg 2017;75(7):1344-1350

3 Mendoza-García LV, Vaillard-Jiménez E, García-Rocha A, Bellot-Arcís C, Paredes-Gallardo V. Effect of orthodontic treatment involving first premolar extractions on mandibular third molar angulation and retromolar space. J Clin Exp Dent 2017;9(3):e333-e337

4 Oenning AC, Neves FS, Alencar PN. Prado RF, Groppo FC, Haiter-Neto F. External root resorption of the second molar associated with third molar impaction: comparison of panoramic radiography and cone beam computed tomography. J Oral Maxillofac Surg 2014;72(8):1444-1455

5 Stanley HR, Alattar M, Collett WK, Stringfellow HR Jr, Spiegel EH. Pathological sequelae of "neglected" impacted third molars. J Oral Pathol 1988;17(3):113-117 
6 Nemcovsky CE, Libfeld H, Zubery Y. Effect of non-erupted 3rd molars on distal roots and supporting structures of approximal teeth. A radiographic survey of 202 cases. J Clin Periodontol 1996;23(9):810-815

7 Selvido DI, Bhattarai BP, Rokaya D, Niyomtham N, Wongsirichat $\mathrm{N}$. Pain in oral and maxillofacial surgery and implant dentistry: types and management. Eur J Dent 2021;15(3): 588-598 doi:10.1055/s-0041-1725212

8 Alrashdan MS, Alkhader M. Psychological factors in oral mucosal and orofacial pain conditions. Eur J Dent 2017;11(4):548-552

9 Yamaoka M, Furusawa K, Ikeda M, Hasegawa T. Root resorption of mandibular second molar teeth associated with the presence of the third molars. Aust Dent J 1999;44(2):112-116
10 van der Linden W, Cleaton-Jones P, Lownie M. Diseases and lesions associated with third molars. Review of 1001 cases. Oral Surg Oral Med Oral Pathol Oral Radiol Endod 1995;79(2):142-145

11 Wang D, He X, Wang Y, et al. External root resorption of the second molar associated with mesially and horizontally impacted mandibular third molar: evidence from cone beam computed tomography. Clin Oral Investig 2017;21(4):1335-1342

12 Potârnichie O, Solomon S, Teslaru S, Pasarin L, Mârtu S. Study on the incidence of external root resorption in current practice. Roman J Oral Rehabil 2012;4(2:72-76

13 Fuss Z, Tsesis I, Lin S. Root resorption-diagnosis, classification and treatment choices based on stimulation factors. Dent Traumatol 2003;19(4):175-182 